\title{
An ESR Investigation of Synthetic Pyrite Crystals
}

\author{
D. Siebert and J. Dahlem \\ Institut für Physikalische Chemie, Universität Freiburg i. Br. \\ S. Fiechter and A. Hartmann \\ Hahn-Meitner-Institut, Berlin \\ Z. Naturforsch. 44a, 59-66 (1989); received November 12, 1988
}

\begin{abstract}
Synthetic pyrite crystals doped with halogen $(\mathrm{Cl}, \mathrm{Br})$ or manganese $(\mathrm{Mn})$ were investigated by ESR at $78 \mathrm{~K}$ with an $\mathrm{X}$-band spectrometer and a $\mathrm{TE}_{102}$ cavity with $100 \mathrm{kc}$ field modulation. The crystals were preferably measured with the directions $\langle 100\rangle,\langle 110\rangle$, and $\langle 111\rangle$ parallel to the static magnetic field $H_{0}$. The ESR spectrum of Mn-doped crystals showed a sextet which can be explained by a spin-Hamiltonian for an electron spin $S=1 / 2$ in interaction with the nuclear spin $I=5 / 2$ of the $\mathrm{Mn}$ nucleus for axial symmetry. Due to the observed spin and due to the orientation of the paramagnetic centers the spectra were assigned to $\mathrm{Mn}^{2+}$ in the low spin state which resides on cation site in the $\mathrm{FeS}_{2}$ lattice. In halogen-doped crystals four pairs of lines can be interpreted by the interaction of the two isotopes of $I=3 / 2,{ }^{79} \mathrm{Br},{ }^{81} \mathrm{Br}$ and ${ }^{35} \mathrm{Cl},{ }^{37} \mathrm{Cl}$, respectively, with an electron spin $S=1 / 2$. According to the observed symmetry of the ESR signals the paramagnetic centers can be explained as $(\mathrm{S}-\mathrm{X})^{2}$ - radicals $(\mathrm{X}=\mathrm{Cl}, \mathrm{Br})$ which substitute for the $\left(\mathrm{S}_{2}\right)^{2-}$ dumb-bells of pyrite. All crystals evoked extremely narrow ESR lines, especially the halogen-doped crystals with linewidths down to $0.03 \mathrm{mT}$. Excellent agreement between measured and calculated spectra up to the finest details has been obtained.
\end{abstract}

Key words: Pyrite, ESR, anorganic radicals, hyperfine coupling, spin densities.

\section{Introduction}

Recently pyrite has attracted great interest due to its photoactive properties that seem to make the material suitable for photoelectrochemical and photovoltaic solar cells $[1,2]$. In natural samples the high impurity concentration disturbs these properties, so that synthetic material has to be applied. The remaining impurities strongly influence the photoactive behaviour, and therefore it is desirable to know the nature of these impurities. If they are paramagnetic, electron spin resonance (ESR) often can be applied with great success.

In spite of the fact that pyrite is one of the best known minerals, there are very few ESR investigations with $\mathrm{FeS}_{2}$ as a host material. In natural crystals the impurity conductivity is mostly too high for ESR measurements, also at low temperatures. Srinivasan et al. [3] reported for the first time ESR spectra of $\mathrm{Mn}^{2+}$ in natural pyrite polycrystals using a $\mathrm{TE}_{101}$ cavity with a variable Gordon coupler to overcome the problems of the considerable dielectric losses. They observed only one hyperfine sextet, but interpreted their results

Reprint requests to Prof. Dr. D. Siebert, Institut für Physikalische Chemie, Albertstr. 21, D-7800 Freiburg. by the usual assumption of a ${ }^{6} \mathrm{~S}_{5 / 2}$ ground state for $\mathrm{Mn}^{2+}$, where five hyperfine sextets are to be expected for a single crystal. Chandler and Bené [4] investigated solid solutions of $\mathrm{Ni}_{x} \mathrm{Fe}_{1-x} \mathrm{~S}_{2}, \mathrm{Co}_{\mathrm{x}} \mathrm{Fe}_{1-\mathrm{x}} \mathrm{S}_{2}$, and $\mathrm{Co}_{x} \mathrm{Ni}_{y} \mathrm{Fe}_{1-x-y} \mathrm{~S}_{2}$ in the range $0.0004<x<0.03$. They used small synthetic crystals, around $1 \mathrm{mg}$ in weight, to prevent cavity loading. This paper reports on an ESR study of larger synthetic single crystals of pyrite enabling us to evaluate the structure of three different paramagnetic centers.

\section{The Crystals}

The Mn-doped crystals were obtained from a Te melt, often as platelets or needles with $\{100\}$ and $\{110\}$ faces parallel to the crystal prism. The growth technique is described in [5]. The crystals were mounted on a suitable inclined plane surface of a quartz rod by vacuum grease. In this manner the important orientations for the static magnetic field $H_{0}$ could be obtained. The most perfect crystals of several $\mathrm{mm}$ in size were grown by a halogen-transport technique described elsewhere [6], using $\mathrm{Cl}$ and $\mathrm{Br}$ as transport agent, furthermore $\mathrm{Cl}$ as a mineralizer for the synthesis from the elements. The polyhedral crystals had ex- 
cellent growth faces, $\{100\},\{111\},\{210\}$, and $\{112\}$, which could be used for crystal orientation by an optical two-circle reflection goniometer. They were glued on a quartz rod with UHU-plus, so that a $\langle 110\rangle$ direction was parallel to the rod, which entered the cavity perpendicular to $H_{0}$. By rotating the rod the most important directions $\langle 100\rangle,\langle 110\rangle$, and $\langle 111\rangle$ could be orientated parallel to $H_{0}$.

The crystal structure of pyrite is well known. It can be considered as an $\mathrm{NaCl}$ structure where the anions are substituted by $\left(\mathrm{S}_{2}\right)^{2-}$ dumb-bells with local $\overline{3}$ axes. The space group is $\mathrm{P} 2_{1} / \mathrm{a} \overline{3}$. The cations' positions together with the centres of the $\mathrm{S}_{2}$ dumb-bells have the local symmetry $\overline{3}$. All these $\overline{3}$ axes are oriented towards the four different $\langle 111\rangle$ directions, alternating arranged in a regular manner.

\section{ESR Spectra and Analysis}

\subsection{Experimental}

The ESR measurements were performed at $78 \mathrm{~K}$ with a conventional Varian X-band spectrometer and a standard $\mathrm{TE}_{102}$ cavity with $100 \mathrm{kc}$ field modulation. At room temperature the cavity loading was too high for the crystal used, but between $78 \mathrm{~K}$ and $90 \mathrm{~K}$ the resonance dip indicated very small cavity losses, a hint at the temperature dependence of the dark conductivity. The microwave frequency was measured with a frequency counter, the magnetic field by a Bruker Hall

Table 1. Classification of $\langle 111\rangle$-oriented paramagnetic centers for three important orientations of the static magnetic field $H_{0}$ with respect to the crystal axes. $N=$ Number of magnetically equivalent centers.

\begin{tabular}{lcc}
\hline Direction of $H_{0}$ & Angle $\theta$ & $N$ \\
\hline$\langle 100\rangle$ & $54.7^{\circ}$ & 4 \\
$\langle 110\rangle$ & 35.3 & 2 \\
& 90.0 & 2 \\
$\langle 111\rangle$ & $0.0^{\circ}$ & 1 \\
& $70.5^{\circ}$ & 3 \\
\hline
\end{tabular}

effect magnetometer, the reading of it was corrected using DPPH.

For a general direction of the magnetic field we observed 24 lines for the Mn-doped crystals and more than fifty lines for the halogen-transported crystals. These numbers were considerably reduced for the $\langle 100\rangle,\langle 110\rangle$, and $\langle 111\rangle$ orientations, indicating that for these directions crystallographically equivalent centers now become also magnetically equivalent. The remaining numbers of lines and their splitting behaviour, if the magnetic field deviates somewhat from these directions, lead us to conclude that there were axial centers, directed to the four different $\langle 111\rangle$ axes. For the special directions mentioned above, the angles $\theta$ between the center axes and $H_{0}$ are given in Table 1 together with the numbers of magnetically equivalent centers.

\subsection{Analysis of the Spectra}

The ESR parameters were obtained from the spectra by a computer fitting procedure that compared experimental and calculated magnetic fields at resonance for an assumed set of ESR parameters. The latter resonance fields were calculated by means of exact numerical diagonalization of the spin-Hamiltonian matrices. For each transition possible in principle, the quantum mechanical transition probability $M_{i j}$ was calculated with the eigenstates $|i\rangle$ and $|j\rangle$ from the diagonalization procedure according to

$$
M_{i j}=\left|\left\langle i\left|\mathscr{H}_{1} / \beta H_{1}\right| j\right\rangle\right|^{2}
$$

with

$$
\begin{aligned}
\left(\mathscr{H}_{1} / \beta H_{1}\right)= & (\cos \theta \cos \phi \cos \chi-\sin \phi \sin \chi) g_{x} S_{x} \\
& +(\cos \theta \sin \phi \cos \chi+\cos \phi \sin \chi) g_{y} S_{y} \\
& -\sin \theta \cos \chi g_{z} S_{z} .
\end{aligned}
$$

Herein $\mathscr{H}_{1}$ denotes the Zeeman operator due to the magnetic field $H_{1}$ of the microwave, the latter with components, expressed by the polar angles $\theta$ and $\phi$ of the static magnetic field $H_{0}$ and the third Eulerian

Table 2. ESR parameters, $g_{N}$ and $Q$ from [14]; energies in $10^{-4} \mathrm{~cm}^{-1}, Q$ in multiples of $|e| \times 10^{-24} \mathrm{~cm}^{2}$; accuracy for the $g$ values: $10^{-4}$, accuracy for the energy parameters: $0.5 \times 10^{-4} \mathrm{~cm}^{-1}$; ' means polarization correction not made.

\begin{tabular}{llllllrrrr}
\hline & $g_{\|}$ & $g_{\perp}$ & \multicolumn{1}{c}{$A_{\|}$} & \multicolumn{1}{c}{$A_{\perp}$} & $P$ & \multicolumn{1}{c}{$A_{0}$} & $T_{\|}$ & $g_{N}$ & $Q$ \\
\hline $\mathrm{Mn}^{2+}(S=1 / 2)$ & 2.0377 & 2.1490 & -8.7 & 75.1 & - & 47.2 & -55.9 & 1.3819 \\
$\left(\mathrm{~S}^{79} \mathrm{Br}\right)^{2-}$ & 2.0344 & 2.0996 & 101.6 & 12.9 & -8.3 & 42.4 & 59.1 & 1.404266 & $0.33^{\text {u }}$ \\
$\left(\mathrm{S}^{81} \mathrm{Br}\right)^{2-}$ & 2.0344 & 2.0996 & 109.6 & 13.6 & -7.0 & 45.6 & 63.9 & 1.513706 & $0.293^{\mathrm{u}}$ \\
$\left(\mathrm{S}^{35} \mathrm{Cl}\right)^{2-}$ & 2.0322 & 2.0839 & 14.8 & 2.4 & 2.0 & 6.5 & 8.3 & 0.547916 & -0.08249 \\
$\left(\mathrm{~S}^{37} \mathrm{Cl}\right)^{2-}$ & 2.0322 & 2.0839 & 12.3 & 1.9 & 1.5 & 5.4 & 6.9 & 0.456082 & -0.06493 \\
\hline
\end{tabular}




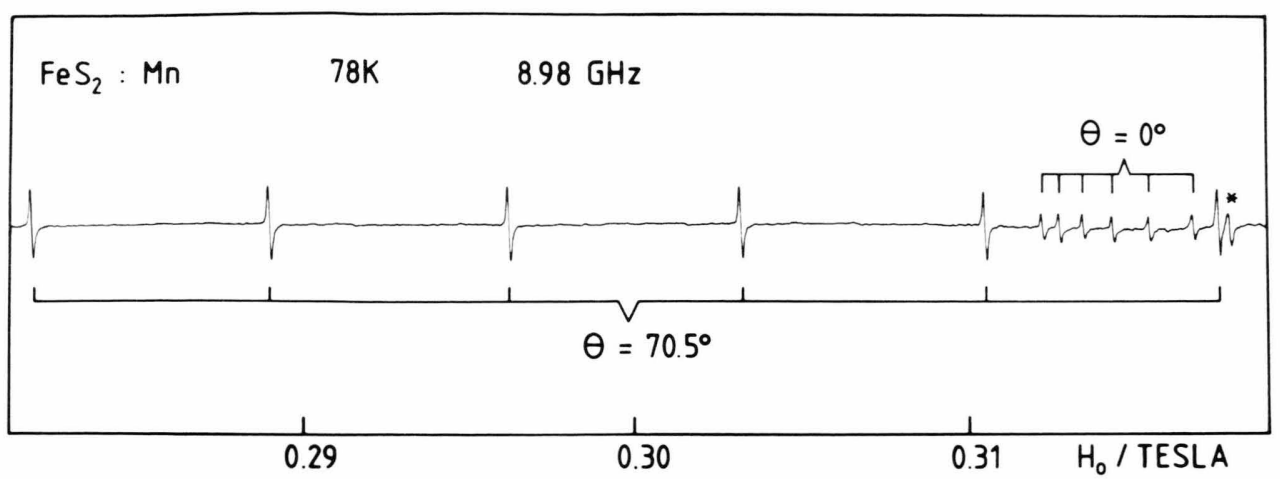

Fig. 1. Experimental and theoretical spectrum for $\mathrm{FeS}_{2}: \mathrm{Mn}^{2+}$ in the low spin state for $H_{0} \|\langle 111\rangle$; * denotes an unidentified signal.

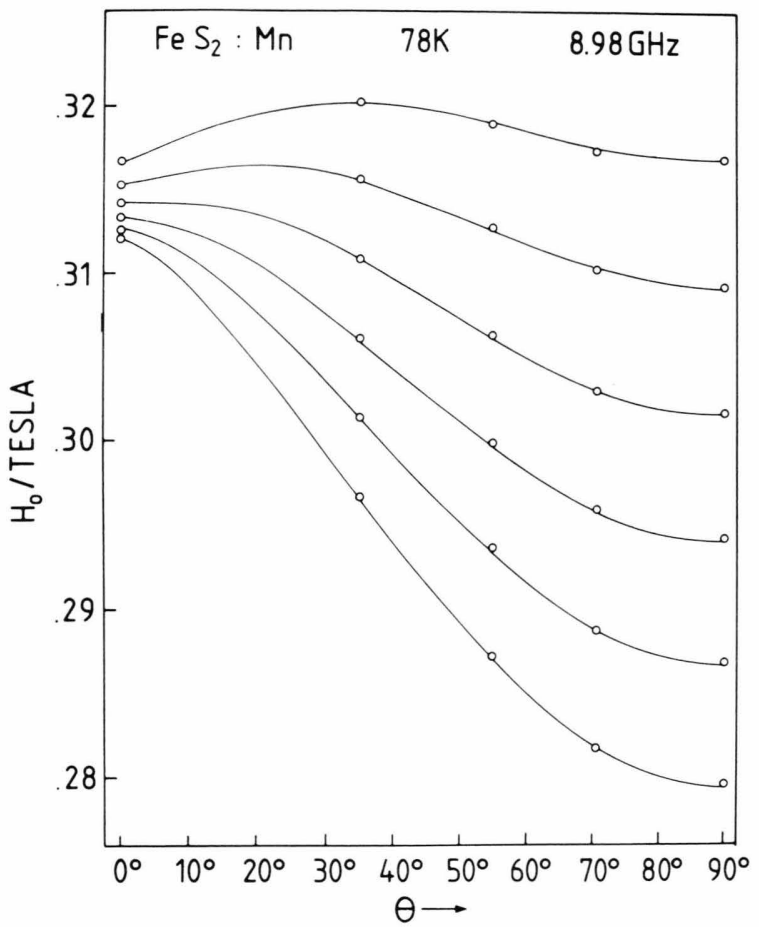

Fig. 2. Angular dependence for $\mathrm{FeS}_{2}: \mathrm{Mn}^{2+}$, 00 measured values.

angle $\chi$ that determines the direction of $H_{1}$. The reference frame is given by the principal axes of the $g$ tensor.

\subsection{Mn-doped Crystals}

The ESR spectrum of an Mn-doped crystal is shown in Fig. 1 for $H_{0} \|\langle 111\rangle$. Corresponding to Table 1, there is a sextet for $\theta=0^{\circ}$ and another one for $\theta=70.5$ with threefold intensity. The surplus line in- dicated in Fig. 1 belongs to an other still unknown impurity, as could be proved by its different temperature dependence. As there were no further side sextets we tried to analyze the spectrum by the following spin-Hamiltonian appropriate for an electron spin $S=1 / 2$ in interaction with the nuclear spin $I=5 / 2$ of the Mn nucleus for axial symmetry:

$$
\begin{aligned}
\mathscr{H}= & g_{\|} \beta H_{z} S_{z}+(1 / 2) g_{\perp} \beta\left(H_{+} S_{-}+H_{-} S_{+}\right) \\
& +A_{\|} S_{z} I_{z}+(1 / 2) A_{\perp}\left(S_{+} I_{-}+S_{-} I_{+}\right) \\
& +P\left(I_{z}^{2}-35 / 12\right) .
\end{aligned}
$$

The matrix representation within the states $|S, M ; I, m\rangle$ $=|1 / 2, M ; 5 / 2, m\rangle(12 \times 12$ matrix $)$ is given in Table 4 (Appendix). As we observed only allowed transitions with $\Delta m=0$, the nuclear Zeeman operator had not to be inculded. The final ESR parameters are summarized in Table 2. From the fitting procedure a value of about $-10^{-4} \mathrm{~cm}^{-1}$ was obtained for $P$, but this value is not very significant and therefore not included in Table 2. It is, however, of the right order of magnitude, the corresponding nuclear quadrupole operator had very little influence on the magnetic fields at resonance. The theoretical stick diagram is also shown in Fig. 1 for $H_{0} \|\langle 111\rangle$, and the angular dependence in Figure 2. It was not possible to reproduce this angular dependence for a middle sextet of a presumptive $S=5 / 2$ spin system. The good agreement of the calculated fields at resonance with the experimental values is a proof that the assumption of an $S=1 / 2$ spin system was right.

\subsection{Halogen Transported Crystals}

In the halogen transported crystals the ESR lines were extremely narrow, about $0.03 \mathrm{mT}$ in width. Thus 


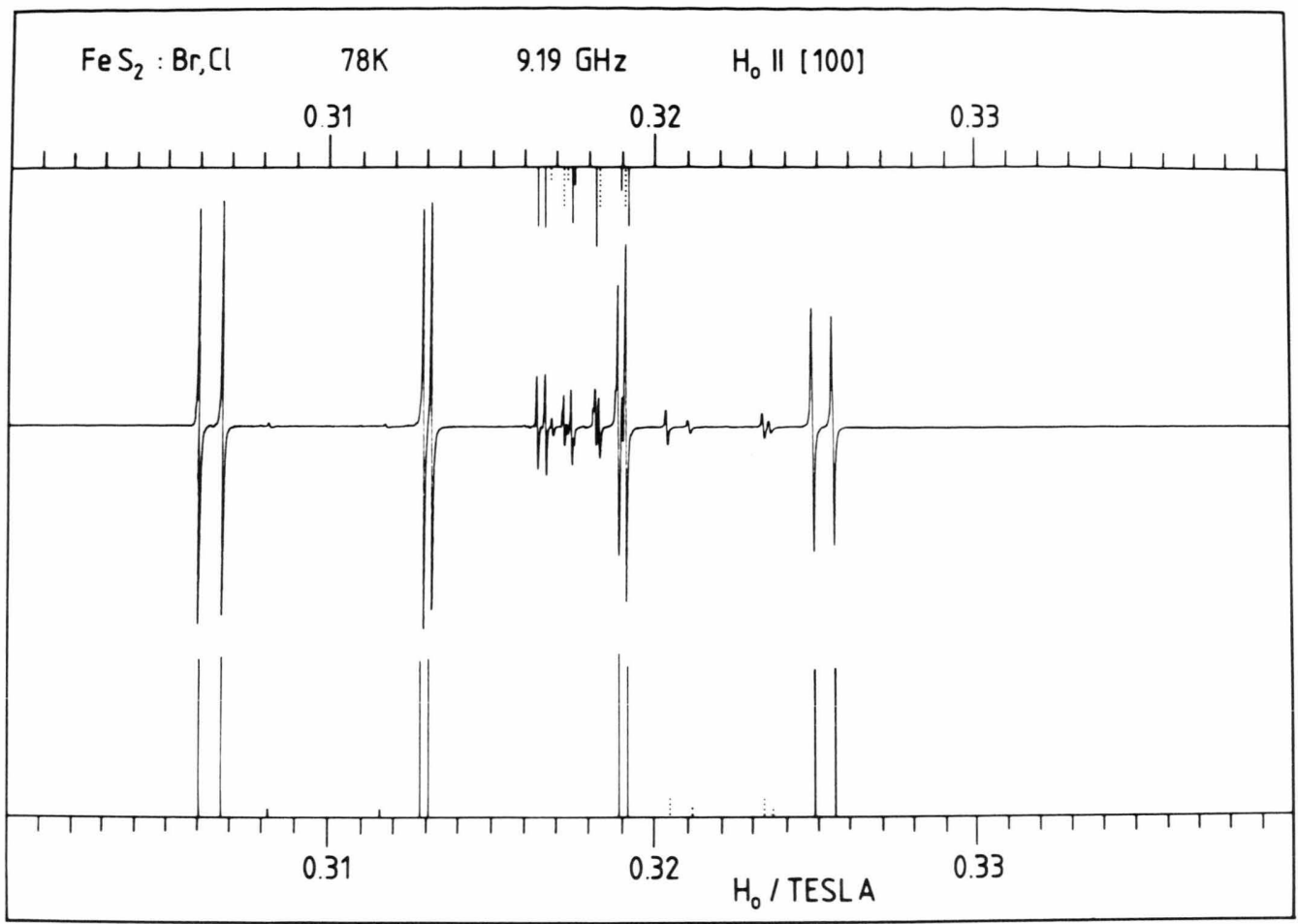

Fig. 3. Experimental and theoretical spectrum for $\mathrm{FeS}_{2}$ : $\mathrm{Br}, \mathrm{Cl}$ with $\mathrm{H}_{0} \|\langle 100\rangle$; upper stick diagram for $\mathrm{FeS}$ : $\mathrm{Cl}_{\text {, lower stick }}$ diagram for $\mathrm{FeS}_{2}: \mathrm{Br}$; dotted sticks: forbidden transitions with $\Delta m \neq 0$.

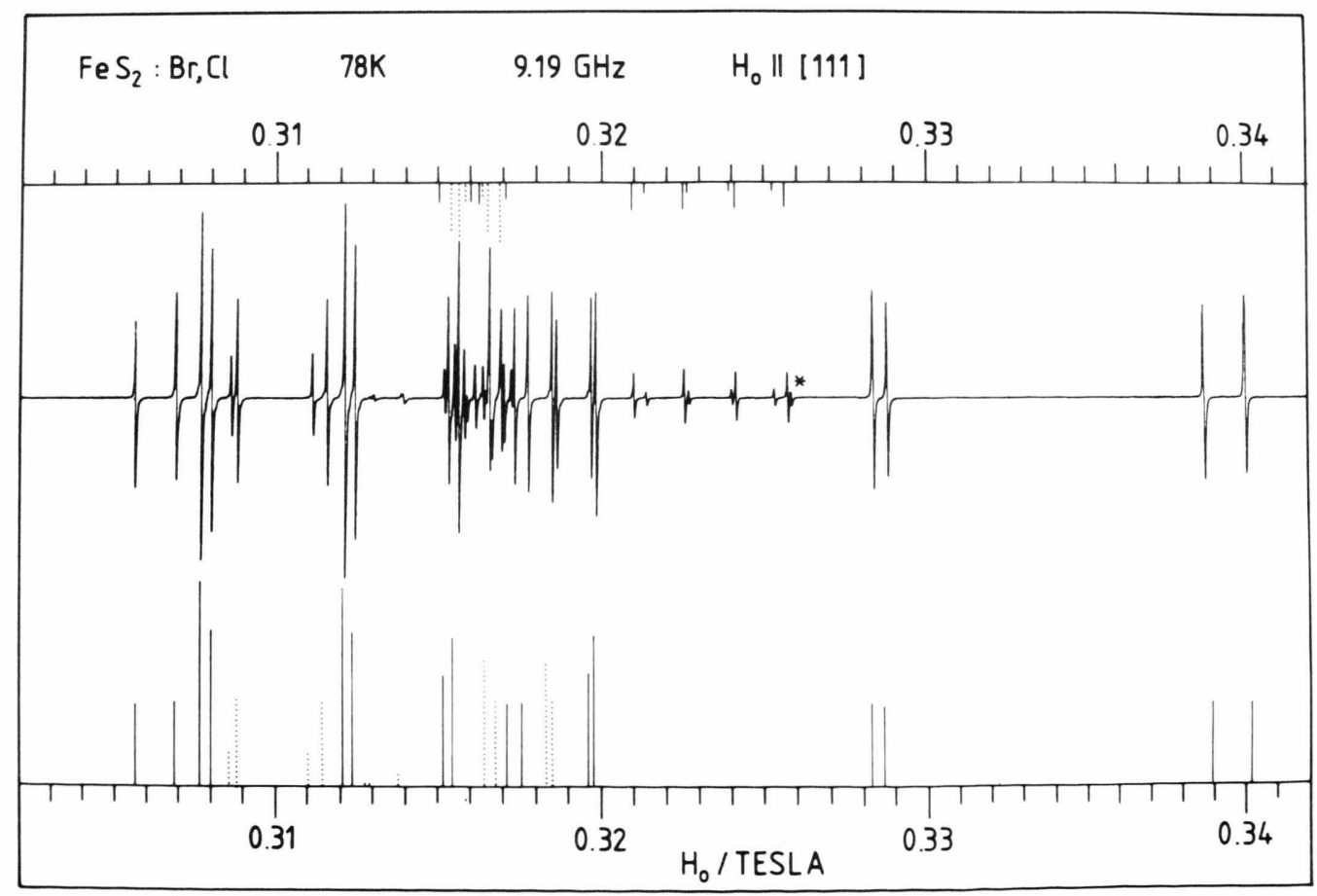

Fig. 4. Experimental and theoretical spectrum for $\mathrm{FeS}_{2}: \mathrm{Br}, \mathrm{Cl}$ with $H_{0} \|\langle 111\rangle$; upper stick diagram for $\mathrm{FeS}$ : $\mathrm{Cl}_{\text {, lower }}$ stick diagram for $\mathrm{FeS}_{2}: \mathrm{Br}$; dotted sticks: forbidden transitions with $\Delta m \neq 0$; * denotes an unidentified signal. 
Table 3. Hyperfine coupling constants and spin densities.

\begin{tabular}{lclll}
\hline & $A_{0}^{*} / \mathrm{MHz}$ & $T_{\|}^{*} / \mathrm{MHz}$ & $\varrho_{\mathrm{s}}$ & $\varrho_{\mathrm{p}}$ \\
\hline$\left(\mathrm{S}^{79} \mathrm{Br}\right)^{2-}$ & 20672 & 1095 & 0.006 & 0.162 \\
$\left(\mathrm{~S}^{35} \mathrm{Cl}\right)^{2-}$ & 4433 & 241 & 0.004 & 0.103 \\
$\left(0^{19} \mathrm{~F}\right)^{2-}$ & - & - & 0.02 & 0.21 \\
\hline
\end{tabular}

their splitting behaviour could be easily observed and the crystals could be very exactly (within 0.1 degree of angle) adjusted for the special directions of $H_{0}$ parallel to $\langle 100\rangle,\langle 110\rangle$, and $\langle 111\rangle$.

The ESR spectrum of a $\mathrm{Br}$-transported crystal is shown in Fig. 3 for $H_{0} \|\langle 100\rangle$ and in Fig. 4 for $H_{0} \|\langle 111\rangle$. In Fig. 3 four pairs of strong lines have nearly the same intensity. If one bears in mind that there are two $\mathrm{Br}$ isotopes of $I=3 / 2$ with nearly the same natural abundance and similar magnetic moments, the strong lines can be explained by $S=1 / 2$ in interaction with the two isotopes of $I=3 / 2,{ }^{79} \mathrm{Br}$ and ${ }^{81} \mathrm{Br}$. In the middle part of the experimental spectrum of Fig. 4 one observes a similar situation, but now one line of each pair is considerably smaller than the other. The intensity ratio is about $3: 1$ and corresponds fairly well to the natural abundance of the two $\mathrm{Cl}$ isotopes, ${ }^{35} \mathrm{Cl}$ and ${ }^{37} \mathrm{Cl}$, that have $I=3 / 2$ too and the abundance ratio 3.01:1. Therefore the spectra had to be described by four spin-Hamiltonians for the different paramagnetic centers with the ${ }^{79} \mathrm{Br},{ }^{81} \mathrm{Br},{ }^{35} \mathrm{Cl}$, and ${ }^{37} \mathrm{Cl}$ isotopes. For the halogen-transported crystals we observed also forbidden lines with $\Delta m \neq 0$, and therefore the nuclear Zeeman operator had to be included. So we analyzed the different line systems with the following spin-Hamiltonian for $S=1 / 2$ and $I=3 / 2$ in axial symmetry:

$$
\begin{aligned}
\mathscr{H}= & g_{\|} \beta H_{z} S_{z}+(1 / 2) g_{\perp} \beta\left(H_{+} S_{-}+H_{-} S_{+}\right) \\
& +A_{\|} S_{z} I_{z}+(1 / 2) A_{\perp}\left(S_{+} I_{-}+S_{-} I_{+}\right) \\
& +P\left(I_{z}^{2}-5 / 4\right)-g_{N} \beta_{N} H_{z} I_{z}-(1 / 2) \\
& \cdot g_{N} \beta_{N}\left(H_{+} I_{-}+H_{-} I_{+}\right) .
\end{aligned}
$$

The corresponding matrix representation within the states $|S, M ; I, m\rangle=|1 / 2, M ; 3 / 2, m\rangle$ is given in Table 5 $(8 \times 8$ matrix $)$. From the fitting procedure the final ESR parameters are listed in Table 2. Because of the high precision of the experimental line positions and the greater number of lines that were fitted, the parameter $P$ is now significant. To demonstrate the agreement between the experimental and calculated ESR lines, the relative heights of the single sticks of the stick diagrams were also calculated and plotted according to (1) and (2) with consideration of the numbers of equivalent centers from Table 1 and the natural abundance of the isotopes. There is excellent agreement between the measured and calculated spectra up to the finest details of forbidden transitions for the three field directions $H_{0}$ parallel to $\langle 100\rangle,\langle 110\rangle$, and $\langle 111\rangle$. This agreement surpasses the accuracy of the plots in Figs. 3 and 4.

\section{Discussion}

A noteworthy characteristic of our spectra is the small linewidth of the observed signals down to $0.03 \mathrm{mT}$ for the halogen transported crystals. Similar linewidths were observed for $\mathrm{Mn}^{2+}$ in $\mathrm{ZnS}(0.04 \mathrm{mT}$ for a line of the middle sextet [7]). A necessary condition for these narrow lines is the absence of nuclear spins of the ions of the host lattice. Apart from this, the crystals must be well ordered and free from internal strains. Viceversa, the extremely narrow signals indicate good crystallinity. We were thus able to determine all the ESR parameters of the Hamiltonian (4).

\section{1. $\mathrm{FeS}_{2}: \mathrm{Mn}$}

Due to the observed spins $S=1 / 2$ and $I=5 / 2$ and due to the orientation of the paramagnetic centers, we assign the spectrum of Fig. 1 to $\mathrm{Mn}^{2+}$ in the low-spin state on cation sites in $\mathrm{FeS}_{2}$. This is in contradiction to Srinivasan et al. [3], who assumed the high-spin configuration but could not determine the angular dependence of Fig. 2 because of the polycrystalline structure of their samples. It is well known that in pyrite $\mathrm{Fe}^{2+}$ is in the low-spin state too [8], and this is apparently transferred to $\mathrm{Mn}^{2+}$. We emphasize this result because in inorganic materials the low-spin configuration is very unusual for $\mathrm{Mn}^{2+}$. For example, in the mineral hauerite $\left(\mathrm{MnS}_{2}\right)$, which also crystallizes in the pyrite structure, $\mathrm{Mn}^{2+}$ occurs exclusively in the high spin state $[9,10]$. After Tanabe and Sugano [11] the low-spin configuration in octahedral symmetry is expected if the crystal field splitting $\Delta=10 \mathrm{Dq}$ surpasses $2 B\left(\mathrm{Fe}^{2+}\right)$ and $2.8 B\left(\mathrm{Mn}^{2+}\right)$, respectively. ( $B$ is the Racah parameter for the corresponding ion.) The latter expression will always be greater than the first one. If one assumes that $\Delta$ does not change very much on substitution of $\mathrm{Fe}^{2+}$ by $\mathrm{Mn}^{2+}$, one can argue that $\Delta$ is at least $2.8 \mathrm{~B}\left(\mathrm{Mn}^{2+}\right)$ for $\mathrm{Fe}^{2+}$ in pyrite. 
The hyperfine interaction tensor $\mathbf{A}$ is decomposed, as usually, in an isotropic part $\mathbf{A}_{0}$ with the principal value $A_{0}$ and a traceless tensor $\mathbf{T}$ with the principal values $T_{\|}$and $T_{\perp}$, whereby $T_{\|}=-2 T_{\perp} \cdot A_{0}$ and $T_{\|}$are included in Table 2.

As we have proved that the $\mathrm{Mn}^{2+}$ center has definitely $S=1 / 2$, we tried to understand the anisotropy of the hyperfine interaction in a simple one electron picture for the unpaired electron. Due to the trigonal distortion, the three $\mathrm{t}_{2 \mathrm{~g}}$ states of $\mathrm{Mn}^{2+}$ in the octahedral complex $\mathrm{Mn}^{2+}\left(\mathrm{S}_{2}{ }^{2-}\right)_{6}$ are further split into one low-lying single level $\left|t_{0}\right\rangle$ and two degenerate higher states $\left|t^{+}\right\rangle$and $\left|t^{-}\right\rangle$. The new orbitals can be expressed by the five normalized $d$ functions $|0\rangle$, $| \pm 1\rangle,| \pm 2\rangle$, related to the trigonal axis as a new axis of quantization [12]:

$$
\begin{aligned}
& \left|t^{+}\right\rangle=(1 / 3)^{1 / 2}|+1\rangle+(2 / 3)^{1 / 2}|-2\rangle, \\
& \left|t^{-}\right\rangle=(1 / 3)^{1 / 2}|-1\rangle-(2 / 3)^{1 / 2}|+2\rangle, \\
& \left|t_{0}\right\rangle=|0\rangle .
\end{aligned}
$$

In the low-spin configuration of $\mathrm{Mn}^{2+}$ these levels are thought to be filled up with the five $d$ electrons so that there remains one unpaired electron of the type $\left|t^{+}\right\rangle$, $\left|t^{-}\right\rangle$. As $\left|t^{+}\right\rangle^{*}=-\left|t^{-}\right\rangle$, both orbitals have the same charge distribution and therefore give rise to the same anisotropic hyperfine splitting. With regard to the trigonal axis we have for the anisotropic hyperfine splitting according to [12]:

$$
\begin{aligned}
T_{\mid} & =g \beta g_{N} \beta_{N}\left\langle\left(3 z^{2}-r^{2}\right) / r^{5}\right\rangle \\
& =g \beta g_{N} \beta_{N}\left\langle 4(\pi / 5)^{1 / 2} Y_{2}^{0} / r^{3}\right\rangle .
\end{aligned}
$$

Here the brackets denote an average over the whole spatial distribution of the unpaired spin. As the $d$ orbitals can also be expressed by the spherical harmonics $Y_{n}^{m}$, it is easy to calculate the mean values through well-known tables for integration. If one uses $\left|t^{+}\right\rangle$or $\left|t^{-}\right\rangle$for the unpaired spin, the result is $-(2 / 7)\left\langle r^{-3}\right\rangle$. With the factor $g \beta g_{N} \beta_{N}$ and $\left\langle r^{-3}\right\rangle$ after [13] we get $-160 \mathrm{MHz}$ for $T$, in excellent agreement with the measured value of $-167 \mathrm{MHz}$ corresponding to $0.00559 \mathrm{~cm}^{-1}$ from Table 2. The isotropic part $A_{0}$ may be explained by a very small admixture of $s$ states. A pure $4 \mathrm{~s}$ electron centred on $\mathrm{Mn}^{2+}$ would produce an isotropic HF constant of $4731 \mathrm{MHz}$ [13] compared to only $141.5 \mathrm{MHz}$ from Table 2 .

A calculation of the principal $g$ values requires a more sophisticated procedure including the orbitals of the ligands too. This is not the aim of this paper.

\subsection{The radicals $(\mathrm{SBr})^{2-}$ and $(\mathrm{SCl})^{2-}$}

According to the interaction of an electron spin $S=1 / 2$ with a single nuclear $\operatorname{spin} I=3 / 2$, and due to the observed symmetry of the ESR signals we assumed two chemically different paramagnetic centers in interaction with a single $\mathrm{Br}$ and $\mathrm{Cl}$, respectively. This is confirmed by the splitting according to natural abundances of the involved halogen isotopes. The ratio of the HF constants for each pair equals the ratio of the nuclear magnetic moments, i.e. the ratio of the $g_{N}$ values. According to Table 2 we have $A_{\|}\left({ }^{81} \mathrm{Br}\right) / A_{\|}\left({ }^{79} \mathrm{Br}\right)$ $=1.079$, in excellent agreement with $g_{N}\left({ }^{81} \mathrm{Br}\right)$ $g_{N}\left({ }^{79} \mathrm{Br}\right)=1.078$ and $A\left({ }^{35} \mathrm{Cl}\right) / A\left({ }^{37} \mathrm{Cl}\right)=1.203 \mathrm{com}-$ pared to $g_{N}\left({ }^{35} \mathrm{Cl}\right) / g_{N}\left({ }^{37} \mathrm{Cl}\right)=1.201$. The centers turned out to be axial, $\langle 111\rangle$-oriented. An assumed single halogen ion would require an unpaired s-electron because of its spin 1/2. But such an s orbital could not produce the large anisotropy of the hyperfine interaction. Therefore we assigned the observed signals to diatomic radicals, $(\mathrm{SBr})^{2-}$ and $(\mathrm{SCl})^{2-}$, respectively. These molecules substitute for the $\left(\mathrm{S}_{2}\right)^{2-}$ dumb-bells, lowering the local symmetry from $\overline{3}$ to 3 that cannot be distinguished by ESR.

Rius et al. [15] observed the similar molecular ion $(\mathrm{OF})^{2-}$ in $\mathrm{MgO}$, and we will follow their discussion to some extend. For the unpaired electron the following linear combination of atomic $\mathrm{s}$ and $\mathrm{p}$ orbitals centred on halogen and sulphur may be adopted:

$$
\begin{aligned}
\psi= & a_{\mathrm{Br}} 4 \mathrm{~s}(\mathrm{Br})+b_{\mathrm{Br}} 4 p_{z}(\mathrm{Br})-a_{\mathrm{S}} 3 \mathrm{~s}(\mathrm{~S}) \\
& +b_{\mathrm{S}} 3 p_{z}(\mathrm{~S}) \text { for }(\mathrm{SBr})^{2-}, \\
\psi= & a_{\mathrm{Cl}} 3 \mathrm{~s}(\mathrm{Cl})+b_{\mathrm{Cl}} 3 p_{z}(\mathrm{Cl})-a_{\mathrm{S}}^{\prime} 3 \mathrm{~s}(\mathrm{~S}) \\
& +b_{\mathrm{S}}^{\prime} 3 p_{z}(\mathrm{~S}) \text { for }(\mathrm{SCl})^{2-},
\end{aligned}
$$

The coefficients $a_{\mathrm{X}}(\mathrm{X}=\mathrm{Br}, \mathrm{Cl})$ determine the isotropic hyperfine interaction with the halogen nucleus in essential, $b_{\mathrm{X}}$ the anisotropic part. The corresponding spin densities $Q$ for the s and $\mathrm{p}$ contributions follow from

$$
\begin{aligned}
& \varrho_{\mathrm{s}}(\mathrm{X})=\left|a_{\mathrm{X}}\right|^{2}=A_{0}(\mathrm{X}) / A_{0}^{*}(\mathrm{X}), \\
& \varrho_{\mathrm{p}}(\mathrm{X})=\left|b_{\mathrm{X}}\right|^{2}=T_{\|}(\mathrm{X}) / T_{\|}^{*}(\mathrm{X}) .
\end{aligned}
$$

The values with an asterisc mean the hyperfine coupling constants of a corresponding electron that would be fully localized on the $\mathrm{X}^{-}$ion. These coupling constants from [13] are given in Table 3 together with the calculated spin densities. For comparison we have included the results for $(\mathrm{OF})^{2-}$ in $\mathrm{MgO}$ from [15]. It is obvious from this Table that the unpaired electron 
of the (SX) $)^{2-}$ molecular ion in $\mathrm{FeS}_{2}$ is mainly localized on the chalcogen site, and that for a larger extend than for $(\mathrm{OF})^{2-}$ in $\mathrm{MgO}$.

Therefore we may indicate the electronic structures of the sulphur radicals as $\mathrm{S}^{-} \mathrm{Br}^{-}$and $\mathrm{S}^{-} \mathrm{Cl}^{-}$, respectively. This is very reasonable due to the tendency of halogens to form inert gas configurations. In consequence, the overall spread of our ESR spectra is considerably smaller than that of the similar spectra due to the well known $\mathrm{FBr}^{-}$and $\mathrm{FCl}^{-}$centers in alkali halides [16], i.e. $0.03 \mathrm{~T}_{\text {for }} \mathrm{S}^{-} \mathrm{Br}^{-}$compared to $0.25 \mathrm{~T}$ for $\mathrm{FBr}^{-}$in $\mathrm{KCl}$. The spreads for the chlorine centers are smaller mainly because of the smaller $g_{N}$ value.

\subsection{Nuclear Quadrupole Interaction}

The quadrupole coupling constant $P$ is proportional to the electric quadrupole moment $Q$ of the nucleus and to the electric field gradient $V_{z z}$. As the latter is identical for isotopes of the same element there should be a proportionality between $P$ and $Q$ for these isotopes. We have from Table $2 P\left({ }^{79} \mathrm{Br}\right) / P\left({ }^{81} \mathrm{Br}\right)$ $=1.19$ whereas the corresponding ratio for the moments $Q$ is 1.09 , furthermore $P\left({ }^{35} \mathrm{Cl}\right) / P\left({ }^{37} \mathrm{Cl}\right)=1.33$ compared to the moment ratio of 1.27 . The corresponding ratios are equal within the experimental errors. In addition, Table 2 indicates a proportionality between $P$ and $Q$ for $\mathrm{Br}$ and $\mathrm{Cl}$, and that means that $V_{z z}$ is about the same for these elements. $V_{z z}$ is the sum of a lattice contribution and an orbital contribution. As the former one will be identical for the different centers, the latter one will be either also the same for the $\mathrm{Br}$ and $\mathrm{Cl}$ centers, or it will be of unimportant magnitude compared to the lattice contribution.

Table 4. Matrix representation for $S=1 / 2$ and $I=5 / 2$ with consideration of hyperfine interaction and nuclear quadrupole interaction; column I contains the matrix elements of the diagonal, column II the elements of the $5^{\text {th }}$ upper parallel, column III the elements of the $6^{\text {th }}$ upper parallel. All the other matrix elements of the upper half are zero.

\begin{tabular}{lll}
\hline I & II & III \\
\hline$b_{\|} n+(5 / 4) A_{\|}+(10 / 3) P$ & 0 & \\
$b_{\| n+(3 / 4)} n-(2 / 3) P$ & $(1 / 2) \sqrt{5} A_{\perp}$ & $b_{\perp} n_{-}$ \\
$b_{\|} n+(1 / 4) A_{\|}-(8 / 3) P$ & & $b_{\perp} n_{-}$ \\
$b_{\|} n-(1 / 4) A_{\|}-(8 / 3) P$ & $(3 / 2) A_{\perp}$ & $b_{\perp} n_{-}$ \\
$b_{\|} n-(3 / 4) A_{\|}-(2 / 3) P$ & & $b_{\perp} n_{-}$ \\
$b_{\|} n-(5 / 4) A_{\|}+(10 / 3) P$ & $(1 / 2) \sqrt{5} A_{\perp}$ & $b_{\perp} n_{-}$ \\
$-b_{\|} n-(5 / 4) A_{\|}+(10 / 3) P$ & 0 & \\
$-b_{\|} n-(3 / 4) A_{\|}-(2 / 3) P$ & & \\
$-b_{\|} n-(1 / 4) A_{\|}-(8 / 3) P$ & & \\
$-b_{\|} n+(1 / 4) A_{\|}-(8 / 3) P$ & & \\
$-b_{\|} n+(3 / 4) A_{\|}-(2 / 3) P$ & & \\
$-b_{\|} n+(5 / 4) A_{\|}+(10 / 3) P$ & & \\
\hline
\end{tabular}

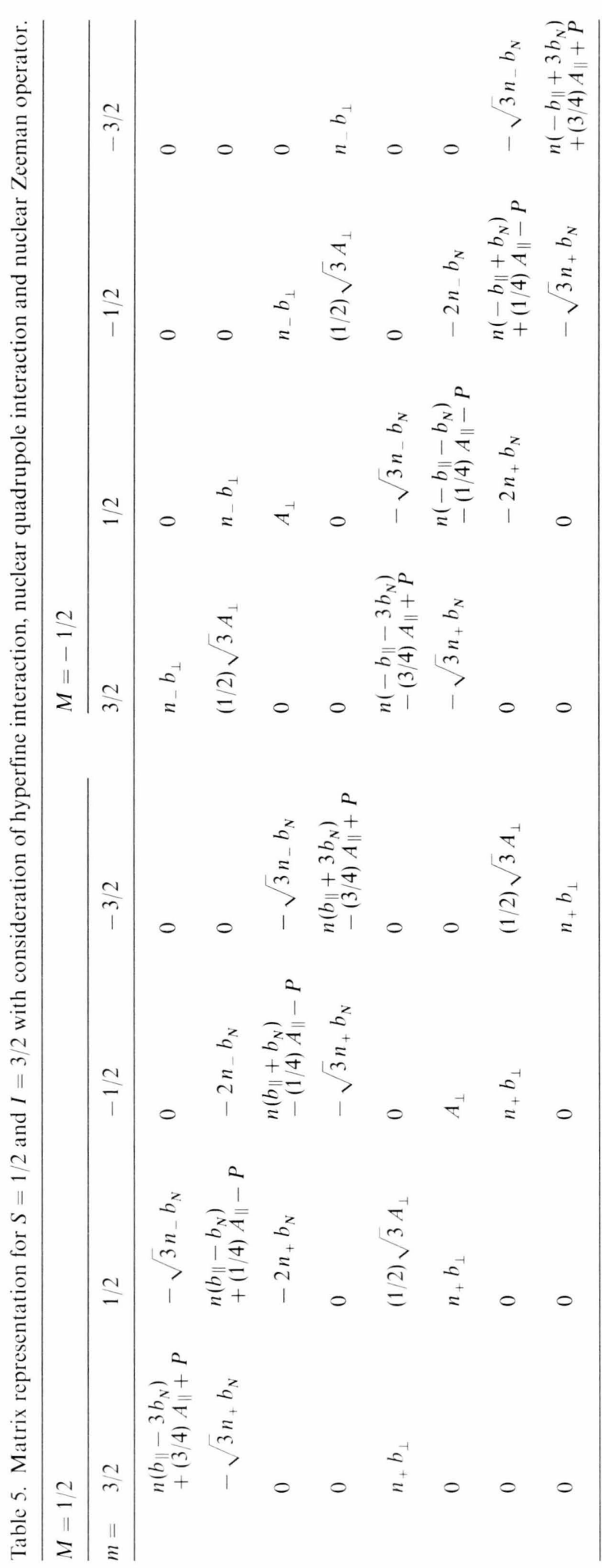




\subsection{Closing Remarks}

In all pyrite samples, natural [17] and synthetic [2], studied so far by photo-electrochemical methods, n-type conductivity was found and often connected with the sulfur deficit of these samples. Our $(\mathrm{S}-\mathrm{X})^{2-}$ defects cannot account for such relatively great sulfur deficits (in the percent region, e.g. $\mathrm{FeS}_{1.94}$ [18]) as their concentration is at least one order of magnitude smaller (e.g. $350 \mathrm{ppm}$ bromine [6]). The hypothesis that $\mathrm{S}^{2-}$ ions replace $\left(\mathrm{S}_{2}\right)^{2-}$ molecules cannot be investigated by ESR directly because $\mathrm{S}^{2-}$ is diamagnetic. Therefore this hypothesis has to be taken into account furtheron. As for the conductivity, we assume that the $(\mathrm{S}-\mathrm{X})^{2-}$ defects act as donors and that this effect becomes more and more important as the other defects, which are responsible for the greater sulfur deficits, diminish.

[1] A. Ennaoui and H. Tributsch, Sol. Cells 13, 197 (1984).

[2] A. Ennaoui, S. Fiechter, W. Jaegemann, and H. Tributsch, J. Electrochem. Soc. 133, 97 (1986).

[3] G. Srinivasan and Mohindar S. Seehra, Solid State Commun. 42, 857 (1982).

[4] R. N. Chandler and R. W. Bené, Physical. Rev. B 8, 4979 (1973).

[5] J. G. Fleming, J. Crystal Growth, in press.

[6] S. Fiechter, J. Mai, A. Ennaoui, and W. Szacki, J. Crystal Growth 78, 438 (1986).

[7] J. Schneider, S. R. Sircar, and A. Räuber, Z. Naturforsch. 18a, 980 (1963).

[8] V. P. Gupta and N. M. Ravindra, Solid State Commun. 32, 1327 (1979).

[9] H. Haraldsen and W. Klemm, Z. Anorg. Allgem. Chemie 223, 409 (1935)

[10] J. B. Goodenough, J. Solid State Chem. 5, 144 (1972).

\section{Acknowlegements}

Financial support of this work by the "Fonds der Chemischen Industrie" and the "Deutsche Forschungsgemeinschaft" is gratefully acknowledged.

\section{Appendix}

For the matrix representations of the Hamiltonians (3) and (4) in Tables 4 and 5 the following abbreviations were used:

$$
\begin{aligned}
& b_{\|}=(1 / 2) g_{\|} \beta H_{0}, \quad b_{\perp}=(1 / 2) g_{\perp} \beta H_{0}, \\
& b_{N}=(1 / 2) g_{N} \beta_{N} H_{0}, \\
& n_{ \pm}=l \pm i m ; l, m, n, \text { are direction cosines, }
\end{aligned}
$$

which determine the direction of the static magnetic field $H_{0}$.

[11] Y. Tanabe and S. Sugano, J. Phys. Soc. Japan 9, 766 (1954).

[12] A. Carrington and A. D. McLachlan, Introduction to Magnetic Resonance, Harper \& Row, New York 1969, p. 155 and 105

[13] A. K. Koh and D. J. Miller, Atomic Data and Nuclear Data Tables, 33, 235 (1985).

[14] Bruker Almanac 1988.

[15] G. Rius, R. T. Cox, P. Freund and J. Owen, J. Phys. C: Solid State Phys. 7, 581 (1974).

[16] D. Schoemaker, Physical Rev. 149, 693 (1966).

[17] R. T. Shuey, Developments in Economic Geology (4), Semiconducting Ore Minerals, Elsevier Scientific Publ. Comp.. Amsterdam 1975

[18] N. Alonso-Vante, G. Chatritheodorou, S. Fiechter, N. Mgoduka, I. Poulios, and H. Tributsch, Solar Energy Materials, in press. 